\title{
Swedish prospective multicenter trial evaluating sentinel lymph node biopsy after neoadjuvant systemic therapy in clinically node-positive breast cancer
}

\author{
Linda Holmstrand Zetterlund ${ }^{1,2}$ (D) Jan Frisell ${ }^{3,4} \cdot$ Athanasios Zouzos $^{5}$ • \\ Rimma Axelsson ${ }^{6,7} \cdot$ Thomas Hatschek $^{8} \cdot$ Jana de Boniface $^{3,9} \cdot$ Fuat Celebioglu $^{1,2}$
}

Received: 12 February 2017/ Accepted: 14 February 2017/Published online: 21 February 2017

(C) The Author(s) 2017. This article is published with open access at Springerlink.com

\begin{abstract}
Purpose Patients with clinically node-positive breast cancer planned for neoadjuvant systemic therapy (NAST) may draw advantages from the nodal downstaging effect and reduce the extent of axillary surgery with sentinel lymph node biopsy (SLNB) performed after NAST. Since there are concerns about lower sentinel lymph node (SLN) detection and higher false-negative rates (FNR) in this setting, our aim was to define the accuracy of SLNB after NAST.

Methods This Swedish national multicenter trial prospectively recruited 195 breast cancer patients from ten hospitals with T1-T4d biopsy-proven node-positive disease planned for NAST between October 1, 2010 and December 31, 2015. Clinically node-negative axillary status after
\end{abstract}

Linda Holmstrand Zetterlund

linda.holmstrand-zetterlund@sll.se

Jan Frisell

jan.frisell@ki.se

Athanasios Zouzos

azouzos@gmail.com

Rimma Axelsson

rimma.axelsson@ki.se

Thomas Hatschek

thomas.hatschek@ki.se

Jana de Boniface

jana.de-boniface@ki.se

Fuat Celebioglu

fuat.celebioglu@sll.se

1 Department of Clinical Science and Education Södersjukhuset, Karolinska Institutet, Stockholm, Sweden

2 Department of Surgery, Södersjukhuset, 11883 Stockholm, Sweden
NAST was not mandatory. SLNB was always attempted and followed by a completion axillary lymph node dissection (ALND).

Results The SLN identification rate was 77.9\% (152/195) but improved to $80.7 \%$ (138/171) with dual mapping. The median number of SLNs was two (range 1-5). A positive SLNB was found in 52\% (79/152), almost 66\% (52/79) of whom had additional positive non-sentinel lymph nodes. The overall pathologic nodal response rate was 33.3\% (66/ $195)$. The overall FNR was $14.1 \%$ (13/92) but decreased to $4 \%(2 / 50)$ when only patients with two or more sentinel nodes were analyzed.

Conclusions In biopsy-proven node-positive breast cancer, SLNB after NAST is feasible even though the identification rate is lower than in clinically node-negative patients.

3 Department of Molecular Medicine and Surgery, Karolinska Institutet, Stockholm, Sweden

4 Departmnet of Breast and Endocrine Surgery, Karolinska University Hospital, 17176 Stockholm, Sweden

5 Department of Radiology, Södersjukhuset, 11883 Stockholm, Sweden

6 Department of Clinical Science, Intervention and Technology, Division of Radiography, Karolinska Institutet, Stockholm, Sweden

7 Department of Radiology, Karolinska University Hospital Huddinge, 14186 Stockholm, Sweden

8 Department of Oncology, Karolinska Institutet, Cancer Center Karolinska and Karolinska University Hospital, 17176 Stockholm, Sweden

9 Department of Surgery, Capio St Göran's Hospital, 11281 Stockholm, Sweden 
Since the overall FNR is unacceptably high, the omission of ALND should only be considered if two or more SLNs are identified.

Keywords Sentinel lymph node biopsy - Breast cancer . Neoadjuvant systemic therapy $\cdot$ False-negative rate . Identification rate $\cdot$ Node-positive

\section{Introduction}

Sentinel lymph node biopsy (SLNB) is today the gold standard nodal staging procedure in clinically node-negative early-stage breast cancer. It is associated with improved staging accuracy and reduced arm morbidity compared with axillary lymph node dissection (ALND) $[1,2]$. In approximately $70 \%$ of early-stage breast cancer patients, SLNB is negative, and ALND can safely be omitted [3]. The safety of SLNB in larger breast tumors has subsequently also been confirmed [4-6].

Locally advanced or inflammatory breast cancer (IBC) planned for neoadjuvant systemic therapy (NAST) implies an increased risk of dissemination to the regional lymph nodes at diagnosis. The standard nodal staging procedure is consequently ALND. Since the indications for NAST have expanded to also encompass operable breast cancer with aggressive tumor biology, the proportion of clinically node-positive patients planned for NAST has decreased [7]. Additionally, current NAST regimens in combination with targeted dual anti-HER2 therapies in HER2-positive patients can achieve nodal downstaging in as many as $70 \%$ of patients $[8,9]$. As a consequence, SLNB was introduced in the neoadjuvant setting.

While SLNB after NAST in clinically node-negative (cN0) patients at diagnosis is associated with high accuracy $[10,11]$, its use in clinically node-positive (cN1) patients is controversial owing to high false-negative rates $[12,13]$. According to the ASCO guidelines from 2014, SLNB may be performed not only in $\mathrm{cN} 0$ patients with operable breast cancer but also in $\mathrm{cN} 1$ patients downstaged to clinical node negativity (ycN0) after NAST; the guidelines advise against performing SLNB in inflammatory breast cancer and do not support SLNB in locally advanced breast cancer due to insufficient data [14]. According to NCCN guidelines from 2016 (version 2.2016), the axilla may be restaged by SLNB after NAST in $\mathrm{cN} 1$ patients at diagnosis if the axilla becomes clinically node-negative after NAST (ycN0); however, marking the biopsied lymph nodes to secure their removal is recommended [15]. Dual mapping is advised to improve the false-negative rate (FNR), which is otherwise higher than $10 \%$ in this subgroup [16]. As the FNR correlates inversely with the number of sentinel lymph nodes retrieved, it is improved in those cases with two or more SLNs identified [17]. In addition, the identification rate (IR) after NAST is lower than for clinically node-negative patients at presentation $[10,18]$ but can be improved with dual mapping [19].

This trial's primary aim was to define the accuracy of SLNB after NAST in a multicenter setting in upfront clinically node-positive patients with $\mathrm{T} 1-4 \mathrm{~d}$ breast cancer.

\section{Methods}

The present Swedish prospective multicenter trial recruited consecutive patients with biopsy-proven invasive $\mathrm{T} 1-4 \mathrm{~d}$ breast cancer planned for NAST from 20 invited hospitals of which 10 actively recruited patients between October 1 , 2010 and December 31, 2015. Ultrasound of the axilla was performed, and if suspicious lymph nodes were encountered sonographically or by physical examination, fineneedle aspiration cytology (FNAC) was performed. Patients were enrolled into two arms depending on their axillary status at presentation.

Clinically N1 patients with biopsy-proven axillary lymph node metastasis were eligible in the here presented arm of the trial which also covered patients with inflammatory breast cancer (IBC). After NAST, SLNB was attempted in all patients together with a completion ALND irrespective of the result of the SLNB. Clinical node negativity after NAST was not a requirement for SLNB to be attempted. Exclusion criteria were allergic reactions to blue dye or radiolabelled colloid, and inability to give informed consent.

Clinically N0 patients had SLNB performed before the start of NAST and will be reported separately.

For more details about the trial, see Clinical.Trials.gov identifier NCT02031042.

\section{Neoadjuvant therapy}

Both neoadjuvant chemotherapy and endocrine therapy were eligible treatments. Standard chemotherapy regimens contained anthracyclines and taxanes, and were given either according to regional guidelines or within ongoing study protocols. Endocrine therapy consisted of aromatase inhibitors. Anti-HER2 therapy was given in combination with taxane-based chemotherapy. Altered or interrupted treatment was recorded together with the reason for disruption.

\section{Response evaluation}

Clinical and radiological response was here evaluated by comparing findings in breast and axillary lymph nodes at diagnosis with those after termination of treatment before 
surgery. Clinical and radiological response was classified according to the UICC criteria [20] apart from radiological partial response which was classified according to the RECIST criteria as more than $30 \%$ decrease in tumor load measured on the greatest diameter [21]. Pathologic response was graded as described by Sataloff et al. evaluating tumor (T) and nodes (N) separately [22] as presented in Table 4.

Post-therapy stage classification (ypTNM) was based on definitions stated in the 7th edition of the AJCC staging system [23]. Pathologic complete response was defined as no residual invasive disease in the breast and axillary lymph nodes (ypT0/is ypN0). The presence of isolated tumor cells (ITC) [ypNO(i+)] was not considered nodal pCR [24].

\section{Lymphatic mapping technique}

Preoperative lymphoscintigraphy was optional. Lymphatic mapping was performed with ${ }^{99 \mathrm{~m}} \mathrm{Tc}$-labeled nanocolloid followed by peroperative use of gamma probe, Patent Blue $\mathrm{V}$ Dye, or both. The magnetic tracer superparamagnetic iron oxide was used alone or in combination with vital blue dye in a few cases. The definition of a sentinel lymph node was the hottest node, any node with more than $10 \%$ of the radioactivity of the hottest node, any blue node, or clinically suspicious nodes on surgical digital exploration.

\section{Pathologic assessment of lymph nodes}

Lymph nodes were handled and assessed according to Swedish National Guidelines for Pathologists. Intraoperative frozen section analysis was not mandatory. All sentinel lymph nodes were fixed in formalin, sliced at $2 \mathrm{~mm}$ intervals, and embedded in paraffin. Each paraffin block was then sectioned at three $200 \mu \mathrm{m}$ levels and each level stained with hematoxylin and eosin. Further staining with cytokeratin if no cancer cells were detected was not mandatory, and was not performed in non-sentinel nodes.

Sentinel lymph node (SLN) metastases were classified according to the 7 th edition of the AJCC breast cancer staging manual [25].

\section{Surgery}

Breast surgery was either breast-conserving surgery or mastectomy. All patients underwent SLNB and a completion axillary dissection of levels I and II.

\section{Definitions}

Clinical tumor stage (cT) was based on pre-NAST radiological size measured by mammography or ultrasound.
The IR was defined as the number of patients with a successfully identified SLN divided by the total number of patients in whom an SLNB was attempted. The FNR was defined as the proportion of patients with a negative SLNB but at least one positive non-sentinel lymph node, divided by all patients with an identified SLNB and at least one positive lymph node after NAST. Accuracy was defined as the proportion of patients with a true-positive or truenegative SLNB out of all patients with successfully identified SLNs.

\section{Statistical analysis}

Continuous variables are presented as median values with their ranges and categorical variables as distributions with their percentages. Comparison of groups according to sentinel lymph node status was performed after exploring normal data distribution. For comparison of non-parametric continuous data, the Mann-Whitney U test was applied. For comparison of non-parametric categorical data, Fisher's exact test was used. A $p$ value of $<0.05$ was considered statistically significant. Sample size calculation was only performed for the cN0 arm, which was the main purpose of this multicenter trial. The statistical software program IBM SPSS Statistics for Windows Version 23.0 (Armonk, NY, USA) was used for all analyzes.

\section{Results}

\section{Patients}

Of 205 eligible patients, 195 patients from ten hospitals operated by 45 surgeons entered the final analysis. A CONSORT diagram is presented in Fig. 1. Median age was 50 years (range 27-84) and median radiological tumor size was $40 \mathrm{~mm}$ (range 11-160). Fifteen patients presented with inflammatory breast cancer (IBC), T4d. The axilla was examined by ultrasound at diagnosis in $99.0 \%$ (193/ 195 ) of patients, and in $98.9 \%$ (191/193), sonographically suspicious lymph nodes were identified. The two patients not examined by axillary ultrasound had suspicious lymph nodes on physical examination. All patients had cytologically confirmed node-positive disease before the initiation of NAST. Clinicopathologic and treatment characteristics are presented in Table 1.

\section{Treatment}

All but one patient received neoadjuvant chemotherapy. The majority had anthracycline plus taxane-based regimens (94.4\%; 184/195). Only one patient in 195 received 


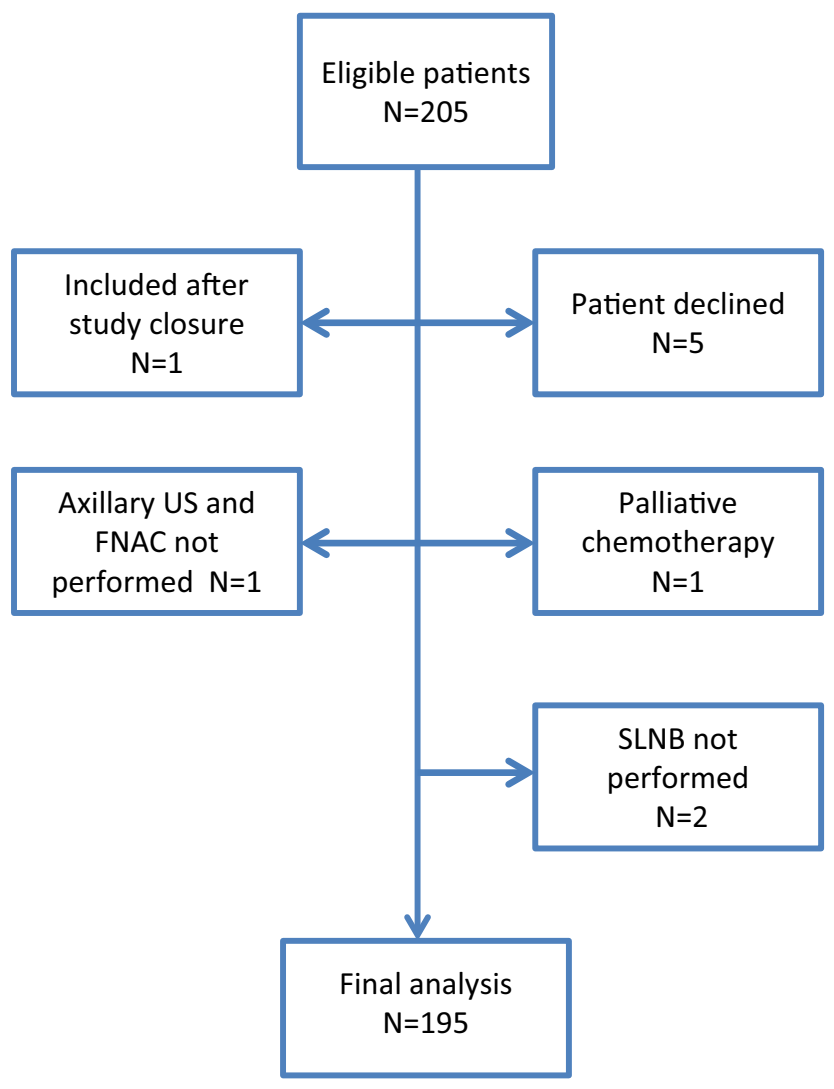

Fig. 1 CONSORT diagram. US ultrasound, FNAC fine needle aspiration cytology, SLNB sentinel lymph node biopsy

neoadjuvant endocrine therapy $(0.5 \%)$. Neoadjuvant systemic therapy regimens are presented in Table 1.

In twelve patients, treatment was interrupted due to intolerable side effects (7), toxicity (2), lack of response (2), or unknown reason (1). In 35 patients, treatment was altered due to intolerable side effects (19), toxicity (9), and lack of response (5); in two patients, a planned shift of therapy was not carried out. Of all HER2-positive patients, 93.5\% (58/62) received targeted treatment, 33.9\% (19/56) of whom received both trastuzumab and pertuzumab. Breast-conserving surgery was performed in 51 of 195 patients $(26.2 \%)$.

\section{SLN detection after NAC}

Lymphatic mapping was performed using a combination of radioisotope and Patent blue dye in $87.5 \%$ (168/192), isotope alone in 5.2\% (10/192), and blue dye alone in 3.6\% (7/ $192)$ of patients. In $3.6 \%$ (7/192), magnetic tracer alone or in combination with blue dye was used.

Overall, at least one SLN was identified in 152 of 195 patients yielding an IR of $77.9 \%$. With dual mapping, regardless of method, the IR was $80.7 \%$ (138/171).
Table 1 Clinicopathologic and treatment characteristics

\begin{tabular}{|c|c|}
\hline & No. $(\%)$ \\
\hline No. of patients & 195 \\
\hline Median years, age & 50 , range $27-84$ \\
\hline \multicolumn{2}{|l|}{ T-stage at presentation } \\
\hline $\mathrm{T} 1$ & $25(12.8)$ \\
\hline $\mathrm{T} 2$ & $94(48.2)$ \\
\hline $\mathrm{T} 3$ & $61(31.3)$ \\
\hline T4d (inflammatory) & $15(7.7)$ \\
\hline \multicolumn{2}{|l|}{ Histological type } \\
\hline Ductal & $158(83.6)$ \\
\hline Lobular & $14(7.4)$ \\
\hline Other & $17(9.0)$ \\
\hline Unknown & $6(3.1)$ \\
\hline \multicolumn{2}{|l|}{ Nottingham histological grade } \\
\hline I & $1(0.7)$ \\
\hline II & $79(55.6)$ \\
\hline III & $62(43.7)$ \\
\hline Unknown & $53(27.2)$ \\
\hline ER-positive & $134(68.7)$ \\
\hline PR-positive & $95(48.7)$ \\
\hline HER2-positive & $62(31.8)$ \\
\hline \multicolumn{2}{|l|}{ Neoadjuvant systemic therapy } \\
\hline Anthracycline plus taxane & $184(94.4)$ \\
\hline Anthracycline only & $7(3.6)$ \\
\hline Other & $3(1.5)$ \\
\hline Aromatase inhibitor & $1(0.5)$ \\
\hline
\end{tabular}

$T$-stage tumor size radiologically, $E R$ estrogen receptor, $P R$ progesteron receptor, HER 2 human epidermal growth factor receptor 2

After excluding patients with IBC, the IR was $79.4 \%$ (143/180), and if dual mapping was employed, it further improved to $82.8 \%$ (130/157).

The median number of retrieved SLNs was two (range 1-5). In 52\% (79/152), SLNB was positive, and in $88.6 \%$ (70/79) of these, at least one macrometastasis was found. The median number of harvested additional axillary lymph nodes was 11 (range 3-41). Fifty-two of 79 (65.8\%) SLNB-positive patients had additional non-sentinel positive lymph nodes. Of all 195 patients, 124 (63.6\%) had residual axillary tumor burden after NAST in either SLNs and/or non-sentinel lymph nodes. The median number of positive nodes was three (range 1-29).

\section{False-negative rate}

Thirteen patients had a negative SLNB after NAST but at least one positive lymph node in non-sentinel lymph nodes yielding an overall FNR of 14.1\% (13/92). A comparison of lymph node status in SLNs and non-sentinel lymph 
Table 2 Comparison of lymph node status in SLNs and overall axillary lymph node status after NAST

\begin{tabular}{llcc}
\hline SLNB & \multicolumn{4}{l}{ Overall axillary nodal status (SLNB and ALND) } \\
\cline { 2 - 4 } & Positive & Negative & Total \\
\hline Positive & 79 & 0 & 79 \\
Negative & 13 & 60 & 73 \\
Total & 92 & 60 & 152 \\
\hline
\end{tabular}

Sensitivity $85.9 \%$ (79/92), specificity $100.0 \%$ (60/60), positive predictive value $100.0 \%$ (79/79), negative predictive value $82.2 \%$ (60/ 73)

SLN sentinel lymph node, SLNB sentinel lymph node biopsy, NAST neaodjuvant systemic therapy, $A L N D$ axillary lymph node dissection

nodes is presented in Table 2. Among the false-negative cases, the median number of positive lymph nodes was 1 (range 1-9) and the median number of retrieved axillary lymph nodes including sentinel lymph nodes was 12 (range 5-20), see Table 3. There were two patients with IBC, and the FNR after excluding these patients was $12.6 \%$ (11/87). False-negative rates calculated for different scenarios are presented in Table 4.

There was no significant difference between patients with a false-negative compared to a true-positive or truenegative SLNB regarding age distribution, tumor size, grade or type, hormone receptor status, HER2 positivity, breast surgery performed, neoadjuvant therapy regimen, anti-HER2-targeted therapy, or proportion of patients with interrupted NAST. However, patients with false-negative SLNs had significantly more altered chemotherapy regimens $(46.2 \%$; 6/13) compared with patients with a true- positive or true-negative SLNB (15.2\%; 21/138; $p=0.013$ ).

\section{Response evaluation}

There was no statistical difference in clinical or radiological response in neither tumor nor lymph nodes between patients with a true-positive or true-negative compared to a false-negative SLNB result. However, there were significantly more patients with a complete/near-complete pathologic response in the tumor (Sataloff A) in the truepositive/true-negative group $(35.3 \%)$ than in the falsenegative group $(7.7 \% ; p=0.044)$, see Table 5 .

Of all patients with an identified SLNB after NAST, $32.9 \%(50 / 152)$ had a complete pathologic response in the breast (ypT0/is), 36.2\% (55/152) a complete pathologic nodal response (ypN0), and 27.6\% (42/152) had an overall complete pathologic response (ypCR). The corresponding figures for all 195 patients were $30.8 \%$ (60/195), 33.3\% (65/195), and 25.6\% (50/195), respectively.

\section{Discussion}

This Swedish multicenter trial evaluates the accuracy of SLNB in the neoadjuvant setting. In the present part of the trial, SLNB was attempted after NAST together with concomitant ALND in $195 \mathrm{~T} 1-4 \mathrm{~d}$ breast cancer patients with biopsy-proven lymph node metastasis at diagnosis. The results of the other part of the same trial regarding $\mathrm{cN} 0$ patients in whom SLNB was performed before and ALND after NAST are reported separately.

Table 3 Thirteen patients with false negative SLNs and corrresponding non-sentinel lymph nodes

\begin{tabular}{|c|c|c|c|c|c|c|}
\hline $\begin{array}{l}\text { Patient } \\
\text { number }\end{array}$ & IBC & $\begin{array}{l}\text { Number of } \\
\text { SLNs }\end{array}$ & $\begin{array}{l}\text { Lymph node status } \\
\text { in SLNs }\end{array}$ & $\begin{array}{l}\text { Number of non-sentinel } \\
\text { lymph nodes }\end{array}$ & $\begin{array}{l}\text { Lymph node status non- } \\
\text { sentinel nodes }\end{array}$ & $\begin{array}{l}\text { Total number of axillary } \\
\text { lymph nodes }\end{array}$ \\
\hline 33 & No & 2 & ypN0 & 7 & ypN1mi & 9 \\
\hline 39 & No & 1 & ypN0 & 9 & ypN1mi & 10 \\
\hline 103 & No & 1 & ypN0 & 5 & ypN1 & 6 \\
\hline 202 & No & 1 & ypN0 & 11 & ypN1(3) & 12 \\
\hline 226 & Yes & 1 & ypN0 & 15 & ypN1mi(7) & 16 \\
\hline 229 & No & 1 & ypN1(i+) & 11 & ypN1 & 12 \\
\hline 232 & No & 1 & ypN0 & 19 & ypN1(9) & 20 \\
\hline 236 & No & 1 & ypN0 & 4 & ypN1 & 5 \\
\hline 292 & No & 1 & ypN0 & 11 & ypN1 & 12 \\
\hline 294 & No & 2 & ypN1(i+) & 6 & ypN1mi & 8 \\
\hline 392 & No & 1 & ypN0 & 14 & ypN1 & 15 \\
\hline 442 & Yes & 1 & ypN0 & 14 & ypN1(6) & 15 \\
\hline 450 & No & 1 & ypN1(i+) & 14 & ypN1 & 15 \\
\hline
\end{tabular}

SLNs sentinel lymph nodes, NAST neoadjuvant systemic therapy, ypN1 macrometastasis, ypN1mi micrometastasis, $y p N 1(i+)$ isolated tumor cells, $I B C$ inflammatory breast cancer 
Table 4 False negative SLN findings after NAST in different scenarios

\begin{tabular}{lllc}
\hline Scenario & True pos $(n)$ & False neg $(n)$ & FNR $^{\mathrm{a}}(\%)$ \\
\hline Overall & 79 & 13 & 14.1 \\
Dual mapping performed & 71 & 11 & 13.4 \\
IBC excluded $(n=15)$ & 76 & 11 & 12.6 \\
ITC considered ypN+ & 87 & 10 & 10.3 \\
SLNB with 1 node retrieved & 31 & 11 & 26.2 \\
SLNB with $\geq 2$ nodes & 48 & 2 & 4.0 \\
SLNB with $\geq 3$ nodes & 23 & 0 & 0.0 \\
\hline
\end{tabular}

${ }^{a}$ Calculated as the number of patients with a false negative SLN in each scenario divided by the number of false negative and true positive SLNs in the same scenario

NAST neoadjuvant systemic therapy, FNR false negative rate, SLN sentinel lymph node, SLNB sentinel lymph node biopsy, IBC inflammatory breast cancer, ITC isolated tumor cells, FNR false negative rate
Table 5 Comparison of response between patients with false-negative to true-positive and true-negative SLNs after NAST

\begin{tabular}{|c|c|c|c|}
\hline & $\begin{array}{l}\text { True-pos and } \\
\text { true-neg }(\%)\end{array}$ & False-neg $(\%)$ & $P$ \\
\hline No. of patients & 139 & 13 & \\
\hline \multicolumn{4}{|c|}{ Pathologic response, tumoral (ypT) } \\
\hline Sataloff T-A & $49(35.3)$ & $1(7.7)$ & \\
\hline Sataloff T-B & $39(28.1)$ & $7(53.8)$ & \\
\hline Sataloff T-C & $43(30.9)$ & $3(23.1)$ & \\
\hline Sataloff T-D & $8(5.8)$ & $2(15.4)$ & 0.044 \\
\hline \multicolumn{4}{|c|}{ Pathological response, nodal (ypN) } \\
\hline Sataloff N-A & $38(27.3)$ & $0(0.0)$ & \\
\hline Sataloff N-B & $22(15.8)$ & $0(0.0)$ & \\
\hline Sataloff N-C & $39(28.1)$ & $5(28.5)$ & \\
\hline Sataloff N-D & $40(28.8)$ & $8(61.5)$ & 0.010 \\
\hline
\end{tabular}

Sataloff T-A: Total or near total therapeutic effect; Sataloff T-B: $>50 \%$ therapeutic effect but less than total or near total; Sataloff T-C: $<50 \%$ therapeutic effect, but effect evident; Sataloff T-D: No therapeutic effect

Sataloff N-A: Evidence of therapeutic effect, no metastatic disease; Sataloff N-B: No nodal metastasis or therapeutic effect; Sataloff N-C: Evidence of therapeutic effect but nodal metastasis still present; Sataloff N-D: Viable metastatic disease, no therapeutic effect

$S L N$ sentinel lymph node, NAST neoadjuvant systemic therapy

The performance of SLNB after NAST in cNO patients at diagnosis is associated with lower IR and higher FNR than SLNB upfront $[10,18]$. The assumed causes for these findings are fibrosis of the lymphatic channels after NAST, altering lymphatic drainage patterns and differential eradication of disease in sentinel and non-sentinel lymph nodes $[26,27]$. In patients with cN1 disease, SLNB after NAST has been questioned because of unacceptably high FNR in some earlier reports [12-14].

The overall IR in the present cohort was $77.9 \%$ (152/ 195). Excluding 15 patients with IBC improved the IR marginally. According to our knowledge, there are only two papers addressing the accuracy of SLNB after NAST in IBC. Both conclude that the method is unreliable in IBC, but base their conclusions on only eight and 20 patients, respectively $[28,29]$. The overall FNR, too, improved in our trial after excluding IBC from analysis. ASCO guidelines from 2014 discourage the performance of SLNB in IBC also after NAST. In case of locally advanced breast cancer downstaged by NAST, data were still considered insufficient to recommend SLNB after NAST [14]. Based on these small studies, we conclude that SLNB after NAST in IBC is feasible but less accurate compared with locally advanced or operable breast cancer, but larger prospective studies are warranted.

An important measure to improve not only the IR but also the FNR is dual mapping, which was recommended but not mandatory in our trial. Dual mapping yielded better overall IR, which is consistent with the prospective SENTINA study reporting an IR of $80.1 \%$ [13]. Also in the NSABP B-27 trial and in ACOSOG Z1071 trial, mapping with radioisotope only or in combination with blue dye was more successful than blue dye alone [19, 27]. In the latter trial, the FNR was significantly reduced by the use of dual mapping [12]. Thus, dual mapping should be the method of choice also in the neoadjuvant setting.

The overall FNR in our trial correlates with the pooled estimate of $15.1 \%$ in a systematic review and meta-analysis evaluating SLNB after NAST in patients with pathologically confirmed node-positive breast cancer. Nijnatten et al. performed a subgroup analysis in which FNR was significantly lower when two or more SLNs were removed [17]. In the ACOSOG Z1071 trial, the FNR decreased from 21.1 to $9.1 \%$ when three or more nodes were examined instead of two [12]. In arm $\mathrm{C}$ of the four-armed prospective SENTINA study, the FNR decreased from $24.3 \%$ if one to $18.5 \%$ if two nodes were removed [13]. Also in our trial, the FNR decreased dramatically when two or more SLNs were retrieved.

A limitation of our trial was that clinical restaging after completion of NAST was not performed. In the ACOSOG Z1071 trial, patients were restaged with axillary ultrasound 
after NAST. Although the reduction of FNR was not statistically significant, sonographically normal lymph nodes correlate with a decreased likelihood of residual nodal disease which can help selecting patients for SLNB after NAST [30].

If all patients with ITC in the SLNs were classified as ypN1 in our study, the overall FNR would have decreased to $10.3 \%$. Since IHC staining was not mandatory, unlike in the SN FNAC study, undiagnosed ITCs are likely. In the SN FNAC study, FNR decreased from $13.3 \%$ when ITC was considered ypN0 to only $8.4 \%$ when $\mathrm{SN}$ metastases of any size were considered positive [31].

In this trial, there was no significant difference in clinical and radiological response between patients with a false-negative compared with a true-positive or true-negative SLN result. The correlation between clinical and pathological response is not reliable related to both the primary tumor and regional lymph nodes [27]. Galimberti et al. retrospectively evaluated $396 \mathrm{cT} 1-4 \mathrm{cN} / \mathrm{cN} 1 / 2$ patients who remained or became ycN0 after NAST. After five years of follow-up, overall survival was not significantly worse in the $\mathrm{cN} 1 / 2$ group. SLN negativity after NAST was a significant predictor of good outcome but only if the breast tumor had responded well [32].

\section{Conclusion}

This trial confirms the feasibility of SLNB after NAST in biopsy-proven node-positive breast cancer at diagnosis. The IR, however, is lower compared with clinically nodenegative patients and the overall FNR is unacceptably high if only one SLN is retrieved. To optimize both IR and FNR, dual mapping should be the method of choice. If only one SLN can be accurately identified and retrieved, a completion ALND should be considered. Pre-NAST marking of the cytologically verified lymph node, selecting only patients with sonographically unsuspicious lymph nodes for SLNB after NAST, and broadening the definition of SLN metastasis after NAST to include isolated tumor cells, all have the potential of further decreasing the FNR.

\footnotetext{
Acknowledgements The authors would like to thank all involved staff at the participating hospitals and their coordinating surgeons and nurses for invaluable help in recruiting study subjects and collecting data: Karolinska University Hospital (J. Frisell, V. Åberg), University Hospital Linköping (C. Hedin), University Hospital Lund (K. Isaksson, C.Ingvar), University Hospital Malmö (L. Rydén), Västmanlands Hospital Västerås (Y.Andersson, L.Bergkvist), University Hospital Örebro (G.Liljegren), Uppsala Academic Hospital (F.Wärnberg), Sahlgrenska University Hospital (R.Olofsson Bagge, S. Janeva), Northern Älvsborg County Hospital (C.Wångblad). We would also like to thank H. Pettersson, biostatistician at Karolinska Institutet, for professional statistical support and pathologist E. Colon for help with response evaluation of neoadjuvant treatment. This research was
}

supported by Grants from the Swedish Breast Cancer Association (BRO), the Stockholm County Council (ALF project, grants 20130403 and 20150097), and Olle Engkvist Byggmästare Foundation. None of them took part neither in the design and conduct of the trial, nor in the preparation, review, approval or decision to submit the manuscript for publication.

\section{Compliance with ethical standards}

Conflict of interest The authors declare that they have no conflict of interest.

Ethical approval All procedures performed in studies involving human participants were in accordance with the ethical standards of the research committee and with the 1964 Helsinki declaration and its later amendments or comparable ethical standards. This trial was approved by the Regional Ethics Committee in Stockholm (2010/44131/4) and the Radiation Protection Committee at Södersjukhuset Stockholm (6/10). Written informed consent was obtained from all individuals before inclusion.

Open Access This article is distributed under the terms of the Creative Commons Attribution 4.0 International License (http://crea tivecommons.org/licenses/by/4.0/), which permits unrestricted use, distribution, and reproduction in any medium, provided you give appropriate credit to the original author(s) and the source, provide a link to the Creative Commons license, and indicate if changes were made.

\section{References}

1. Giuliano AE, Dale PS, Turner PR, Morton DL, Evans SW, Krasne DL (1995) Improved axillary staging of breast cancer with sentinel lymphadenectomy. Ann Surg 222(3):394-399 discussion 9-401

2. Mansel RE, Fallowfield L, Kissin M, Goyal A, Newcombe RG, Dixon JM et al (2006) Randomized multicenter trial of sentinel node biopsy versus standard axillary treatment in operable breast cancer: the ALMANAC Trial. J Natl Cancer Inst 98(9):599-609

3. Krag DN, Anderson SJ, Julian TB, Brown AM, Harlow SP, Costantino JP et al (2010) Sentinel-lymph-node resection compared with conventional axillary-lymph-node dissection in clinically node-negative patients with breast cancer: overall survival findings from the NSABP B-32 randomised phase 3 trial. Lancet Oncol 11(10):927-933

4. Bedrosian I, Reynolds C, Mick R, Callans LS, Grant CS, Donohue $\mathrm{JH}$ et al (2000) Accuracy of sentinel lymph node biopsy in patients with large primary breast tumors. Cancer 88(11):2540 2545

5. Chung MH, Ye W, Giuliano AE (2001) Role for sentinel lymph node dissection in the management of large $(>$ or $=5 \mathrm{~cm})$ invasive breast cancer. Ann Surg Oncol 8(9):688-692

6. Schule J, Frisell J, Ingvar C, Bergkvist L (2007) Sentinel node biopsy for breast cancer larger than $3 \mathrm{~cm}$ in diameter. Br J Surg 94(8):948-951

7. Fisher B, Brown A, Mamounas E, Wieand S, Robidoux A, Margolese RG et al (1997) Effect of preoperative chemotherapy on local-regional disease in women with operable breast cancer: findings from National Surgical Adjuvant Breast and Bowel Project B-18. J Clin Oncol 15(7):2483-2493

8. Gianni L, Pienkowski T, Im YH, Roman L, Tseng LM, Liu MC et al (2012) Efficacy and safety of neoadjuvant pertuzumab and trastuzumab in women with locally advanced, inflammatory, or early HER2-positive breast cancer (NeoSphere): a randomised multicentre, open-label, phase 2 trial. Lancet Oncol 13(1):25-32 
9. Schneeweiss A, Chia S, Hickish T, Harvey V, Eniu A, Hegg R et al (2013) Pertuzumab plus trastuzumab in combination with standard neoadjuvant anthracycline-containing and anthracycline-free chemotherapy regimens in patients with HER2-positive early breast cancer: a randomized phase II cardiac safety study (TRYPHAENA). Ann Oncol 24(9):2278-2284

10. Classe JM, Bordes V, Campion L, Mignotte H, Dravet F, Leveque $J$ et al (2009) Sentinel lymph node biopsy after neoadjuvant chemotherapy for advanced breast cancer: results of Ganglion Sentinelle et Chimiotherapie Neoadjuvante, a French prospective multicentric study. J Clin Oncol 27(5):726-732

11. Hunt KK, Yi M, Mittendorf EA, Guerrero C, Babiera GV, Bedrosian I et al (2009) Sentinel lymph node surgery after neoadjuvant chemotherapy is accurate and reduces the need for axillary dissection in breast cancer patients. Ann Surg 250(4):558-566

12. Boughey JC, Suman VJ, Mittendorf EA, Ahrendt GM, Wilke LG, Taback B et al (2013) Sentinel lymph node surgery after neoadjuvant chemotherapy in patients with node-positive breast cancer: the ACOSOG Z1071 (Alliance) clinical trial. JAMA 310(14):1455-1461

13. Kuehn T, Bauerfeind I, Fehm T, Fleige B, Hausschild M, Helms G et al (2013) Sentinel-lymph-node biopsy in patients with breast cancer before and after neoadjuvant chemotherapy (SENTINA): a prospective, multicentre cohort study. Lancet Oncol 14(7):609-618

14. Lyman GH, Temin S, Edge SB, Newman LA, Turner RR, Weaver DL et al (2014) Sentinel lymph node biopsy for patients with early-stage breast cancer: american Society of Clinical Oncology clinical practice guideline update. J Clin Oncol 32(13): 1365-1383

15. Caudle AS, Yang WT, Krishnamurthy S, Mittendorf EA, Black DM, Gilcrease MZ et al (2016) Improved Axillary Evaluation Following Neoadjuvant Therapy for Patients With Node-Positive Breast Cancer Using Selective Evaluation of Clipped Nodes: implementation of Targeted Axillary Dissection. J Clin Oncol 34(10):1072-1078

16. Network NCC. NCCN Clinical Practice Guidelines in Oncology, Version 2.2016 Invasive Breast Cancer. 2016

17. van Nijnatten TJ, Schipper RJ, Lobbes MB, Nelemans PJ, BeetsTan RG, Smidt ML (2015) The diagnostic performance of sentinel lymph node biopsy in pathologically confirmed node positive breast cancer patients after neoadjuvant systemic therapy: a systematic review and meta-analysis. Eur J Surg Oncol 41(10):1278-1287

18. van der Heiden-van der Loo M, de Munck L, Sonke GS, van Dalen T, van Diest PJ, van den Bongard HJ et al (2015) Population based study on sentinel node biopsy before or after neoadjuvant chemotherapy in clinically node negative breast cancer patients: Identification rate and influence on axillary treatment. Eur J Cancer 51(8):915-921

19. Boughey JC, Suman VJ, Mittendorf EA, Ahrendt GM, Wilke LG, Taback B et al (2015) Factors affecting sentinel lymph node identification rate after neoadjuvant chemotherapy for breast cancer patients enrolled in ACOSOG Z1071 (Alliance). Ann Surg 261(3):547-552
20. Miller AB, Hoogstraten B, Staquet M, Winkler A (1981) Reporting results of cancer treatment. Cancer 47(1):207-214

21. Eisenhauer EA, Therasse P, Bogaerts J, Schwartz LH, Sargent D, Ford R et al (2009) New response evaluation criteria in solid tumours: revised RECIST guideline (version 1.1). Eur J Cancer 45(2):228-247

22. Sataloff DM, Mason BA, Prestipino AJ, Seinige UL, Lieber CP, Baloch Z (1995) Pathologic response to induction chemotherapy in locally advanced carcinoma of the breast: a determinant of outcome. J Am Coll Surg 180(3):297-306

23. Edge SB, Compton CC (2010) The American Joint Committee on Cancer: the 7th edition of the AJCC cancer staging manual and the future of TNM. Ann Surg Ocol 17(6):1471-1474

24. Bossuyt V, Provenzano E, Symmans WF, Boughey JC, Coles C, Curigliano $G$ et al (2015) Recommendations for standardized pathological characterization of residual disease for neoadjuvant clinical trials of breast cancer by the BIG-NABCG collaboration. Ann Oncol 26(7):1280-1291

25. AJCC. Breast cancer staging AJCC 7th edition: American Joint Committee on Cancer 2009. https://cancerstaging.org/referencestools/quickreferences/pages/default.aspx. Accessed on July 2016

26. Jones JL, Zabicki K, Christian RL, Gadd MA, Hughes KS, Lesnikoski BA et al (2005) A comparison of sentinel node biopsy before and after neoadjuvant chemotherapy: timing is important. Am J Surg 190(4):517-520

27. Mamounas EP, Brown A, Anderson S, Smith R, Julian T, Miller B et al (2005) Sentinel node biopsy after neoadjuvant chemotherapy in breast cancer: results from National Surgical Adjuvant Breast and Bowel Project Protocol B-27. J Clin Oncol 23(12):2694-2702

28. Hidar S, Bibi M, Gharbi O, Tebra S, Trabelsi A, Korbi S et al (2009) Sentinel lymph node biopsy after neoadjuvant chemotherapy in inflammatory breast cancer. Int J Surg 7(3):272-275

29. Stearns V, Ewing CA, Slack R, Penannen MF, Hayes DF, Tsangaris TN (2002) Sentinel lymphadenectomy after neoadjuvant chemotherapy for breast cancer may reliably represent the axilla except for inflammatory breast cancer. Ann Surg Oncol 9(3):235-242

30. Boughey JC, Ballman KV, Hunt KK, McCall LM, Mittendorf EA, Ahrendt GM et al (2015) Axillary ultrasound after neoadjuvant chemotherapy and its impact on sentinel lymph node surgery: results from the American College of Surgeons Oncology Group Z1071 Trial (Alliance). J Clin Oncol 33(30):3386-3393

31. Boileau JF, Poirier B, Basik M, Holloway CM, Gaboury L, Sideris L et al (2015) Sentinel node biopsy after neoadjuvant chemotherapy in biopsy-proven node-positive breast cancer: the SN FNAC study. J Clin Oncol 33(3):258-264

32. Galimberti V, Ribeiro Fontana SK, Maisonneuve P, Steccanella F, Vento AR, Intra M et al (2016) Sentinel node biopsy after neoadjuvant treatment in breast cancer: five-year follow-up of patients with clinically node-negative or node-positive disease before treatment. Eur J Surg Oncol 42(3):361-368 